\title{
Evolución del uso de los materiales plásticos en la industria automotriz
}

\section{Evolution of the use of plastic materials in the automotive industry}

\author{
Virgilio Lucas Ramos Rivero \\ Universidad Internacional del Ecuador, Ecuador
}

Autor para correspondencia: viramosri@uide.edu.ec

Fecha de recepción: 20 de agosto de 2018 Fecha de aceptación: 15 noviembre del 2018

Resumen: El desarrollo de la industria automotriz está relacionado directamente y de forma significativa al uso de diferentes materiales y aunque no todos los autos son iguales, los materiales de que se construyen son prácticamente los mismos. La satisfacción de los requerimientos propios de un auto, los costos de producción, la disminución de costos relacionados con el consumo de combustible, la disminución del peso y la seguridad, son factores esenciales para el diseño y la selección de los materiales apropiados y su combinación. Entre los materiales que de manera progresiva y contundente está siendo utilizado en la fabricación de automóviles se encuentran los polímeros o plásticos, que dadas sus propiedades atractivas para satisfacer algunos de los requisitos de diferentes componentes y cualidades de un auto, están marcando la diferencia en algunos aspectos en relación con otros materiales. El presente artículo brinda información sucinta acerca de la evolución del uso de los materiales polímeros o plásticos en la industria automotriz, las ventajas que estos presentan con relación a otros materiales, sus diferentes tipos, su incidencia en el diseño de automóviles y otros aspectos de interés.

Palabras Clave: evolución; materiales plásticos; industria automotriz

\begin{abstract}
The development of the automotive industry is directly and significantly related to the use of different materials and although not all cars are the same, the materials that they are built are practically the same. The satisfaction of the requirements of a car, the costs of production, the reduction of costs related to fuel consumption, the reduction of weight and safety, are essential factors for the design and selection of the appropriate materials and their combination. Among the materials that are being used progressively and forcefully in the manufacture of automobiles are polymers or plastics, which due to their attractive properties to meet some of the requirements of different components and qualities of a car, are making a difference in some aspects in relation to other materials. This article provides succinct information about the evolution of the use of polymer or plastic materials in the automotive industry, the advantages they present in relation to other materials, their different types, their impact on the design of automobiles and other aspects of interest.
\end{abstract}

Key words: evolution; plastic materials; automotive industry 


\section{Introducción}

A partir de las primeras versiones de autos que hoy nos parecen quizás un tanto rudimentarias y simples pero que revolucionaron la industria, mejoraron sustancialmente el estilo de vida de las personas y aceleraron por así decirlo, el ritmo de desarrollo de la humanidad, estos han continuado evolucionando de manera vertiginosa en el transcurso de los años, haciéndose cada vez más estilizados, lujosos, cómodos, versátiles, livianos y seguros.

Para el logro de las transformaciones señaladas, se han desarrollado nuevas tecnologías con un carácter verdaderamente innovador que incluyen la producción e implementación de diversos materiales y su combinación, donde ya juegan un importante papel los polímeros o plásticos.

Basado en el estudio de diferentes fuentes bibliográficas y la experiencia del autor el presente artículo tiene como objetivo brindar de una manera sucinta, información acerca de la evolución del uso de los materiales en la industria automotriz, con énfasis en los polímeros o plásticos. Se destacan las propiedades de estos últimos y las ventajas que presentan con relación a otros materiales en la solución de determinados problemas técnicos, sus diferentes tipos, su incidencia en el diseño de automóviles y otros aspectos de interés.

\section{Materiales y Métodos}

Para el desarrollo del presente trabajo se realizó una investigación en diferentes fuentes de información documental acerca del uso de los diferentes materiales en la industria automotriz y se tuvieron en cuenta las experiencias del autor en su actividad como docente en la cátedra de Tecnología de Materiales, en la carrera de Ingeniería Automotriz que se desarrolla en Universidad Internacional del Ecuador, sede Guayaquil. Se tomaron como referencias apuntes del autor y resúmenes realizados por este acerca de materiales utilizados en la rama automotriz. Se emplearon básicamente métodos de carácter teórico, como son el método histórico lógico, el analítico sintético y el inductivo deductivo.

\section{Antecedentes}

\section{Fundamentación}

Desde el surgimiento del automóvil hasta la fecha, su construcción se ha sustentado en líneas generales en el empleo de materiales metálicos y sus aleaciones, siendo entre ellos el acero, la aleación más utilizada en la historia de la industria automotriz. Dicha preferencia se debe a las excelentes cualidades de esta aleación de hierro con carbono, comenzando con su alta resistencia mecánica, dureza y confortabilidad, unido a la abundancia de hierro en la naturaleza y su producción económica.

La elevada resistencia del acero lo convirtió en un material muy conveniente para soportar las cargas que actúan en la estructura del vehículo y de otros elementos de máquina que lo constituyen, lo cual, unido a la tendencia inicial de lograr una alta durabilidad del automóvil, propiciaban la utilización de grandes cantidades de metales, particularmente de acero. (Muñoz, 1998) 
Sin embargo, una de las desventajas del acero, resulta ser su alta densidad, lo que hace que esto influya notablemente en el aumento del peso del vehículo y de la misma forma en el incremento de su consumo de combustible. Por otro lado, durante muchos años se vió en parte comprometida la seguridad de los conductores debido a la rigidez de los parachoques construidos de acero, Esto se debe a que en caso de un choque frontal, una defensa metálica tan solo se doblaría, pero arrastrando con ella los anclajes que la sujetan al chasís, la parrilla, el radiador, el motor y demás componentes, lo cual compromete la vida de los ocupantes.

El comportamiento en la fabricación de automóviles descrito anteriormente se manifestó por un tiempo prolongado, hasta que, en los años 70, al producirse una de las mayores crisis energéticas en el planeta, las tendencias en la industria automotriz se modificaron. Cambios radicales en el diseño y producción de los vehículos automotores condujeron a una mejoría en los procesos de combustión y a una reducción en el peso de los vehículos, lo cual a su vez condujo a una considerable disminución en el empleo de combustible. (A.G.V.A.G., 2002)

Si bien es cierto que aún el uso del acero, el aluminio y otros metales siguen estando presentes en partes estructurales y componentes de un automóvil, las propias necesidades de los usuarios y el proceso de desarrollo de la industria de automoción han estimulado el empleo progresivo de otros materiales en la construcción de automóviles, entre los que se destaca el uso de los materiales plásticos. La palabra plásticos se empleó por primera vez como sustantivo en 1909 y suele emplearse como sinónimo de polímeros (Schmidt, 2008).

\section{Ventajas del empleo de los plásticos en los vehículos}

Las principales razones que han llevado a los fabricantes de automóviles a incorporar plásticos de forma masiva han sido:

- La disminución del peso, que puede oscilar del 17 al 50\%, consiguiendo con ello aumentar las prestaciones finales del vehículo.

- Una mayor resistencia a la fricción (cojinetes y casquillos).

- Absorción de energía durante un impacto sin deformarse dependiendo por supuesto de la magnitud de este (parachoques y otros elementos de carrocerías).

- Resistencia al efecto de productos químicos y la corrosión (depósitos de combustible y de expansión del circuito de refrigeración), entre otros.

- Posibilidad de ser pintados.

- La posibilidad de combinar con otros materiales para mejorar la estética del vehículo.

- Alta moldeabilidad, que permite conseguir piezas variadas y complejas.

- Buenas propiedades de aislamiento térmico, eléctrico y acústico. (A. C., 2009)

Los inicios en el uso de los plásticos en la producción de automóviles Puede decirse que los plásticos han sido utilizados como uno de los materiales para la producción de automóviles desde los inicios del siglo pasado y que en la misma medida que la industria del plástico se fue desarrollando, se fue ampliando progresivamente el uso de este en el sector automotriz.

Un elemento importante en el desarrollo de los plásticos lo constituyó la nitrocelulosa como una de las más antiguas resinas sintéticas empleadas en la fabricación de pinturas y barnices de alto desempeño; dicha resina se fabricó por primera vez hace más de 160 años y pasó a ser ampliamente empleada en pinturas inmediatamente después de finalizada la 1.a Guerra 
Mundial, siendo la responsable de la popularización de las lacas automovilísticas e industriales. Debido a su rápido secado y alto desempeño, se convirtió en el factor principal de la producción en masa de los automóviles (Nitro Química, 2018).

Lo anterior muestra como desde un inicio el uso de los materiales sintéticos se convirtió en un potencial aliado de la fabricación de automóviles, como elemento de protección anticorrosivo y por otro lado estético, pero ampliando progresivamente su participación en el desarrollo de la industria automovilística.

Es en 1907 cuando se introducen los polímeros sintéticos, cuando el Dr. Leo Baeckeland descubre un compuesto de fenol-formaldehído al cual denomina "baquelita" y que se comercializa en 1909. Este material presenta gran resistencia mecánica aislamiento eléctrico y resistencia a elevadas temperaturas (Condezo, 2018).

Con este producto, la industria eléctrica en expansión tuvo un desarrollo formidable, porque la baquelita -como todos los plásticos- es un pésimo conductor y por lo tanto un aislante excelente. Enchufes, manijas, interruptores se empezaron a hacer de este material.

Por otro lado, en los primeros automóviles construidos, las láminas de nitrocelulosa fueron utilizadas regularmente en la fabricación de volantes y componentes eléctricos.

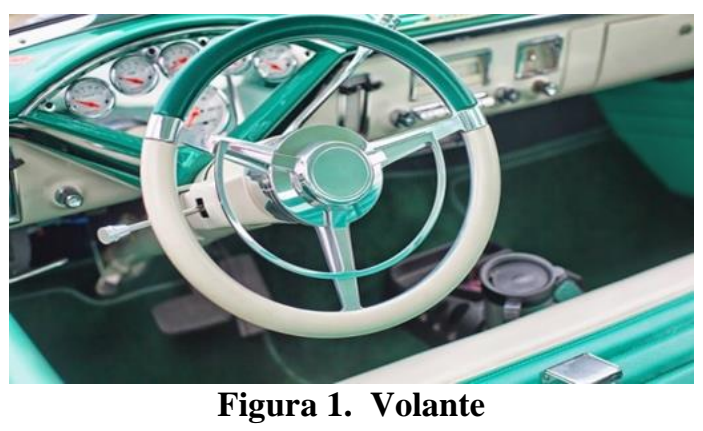

En la década de los 50 el desarrollo de investigaciones para el mejoramiento de las propiedades de los plásticos dio origen a la producción en cantidades considerables de termoplásticos con altas prestaciones ingenieriles. De esta forma se crea El ABS, la Poliamida (PA), el poliacetal (POM) y el policarbonato (PC). La obtención de combinaciones de diferentes plásticos constituyó un salto cualitativo en el desarrollo de estos materiales, lográndose como resultado el surgimiento del poliestireno (PS) como una modificación del óxido polifenileno. En los años 60, se destaca el desarrollo de composites termoestables para el sector automovilístico, primero se aplicó a cuerpos completos monocasco, fabricados con resinas de poliéster reforzados con fibras de vidrio, y posteriormente con mezclas de poliéster y vidrio, que pudieran ser moldeadas por compresión y utilizando una forma de inyección conocidas como la tecnología de Sheet Moulding Compound y la Bulk Moulding Compound. Es sobre todo en los años 70 que, gracias a desarrollo de los polímeros y a las formulaciones adecuadas, los plásticos empezaron a llegar a las partes más significativas del automóvil. En este sentido, el primer terreno en que se imponen es en el parachoques, que es rediseñado completamente (con un efecto que repercute sobre la imagen entera del coche y se convierte en un subsistema del coche distinto formal y funcionalmente del componente de partida (Ezio, 1993). 


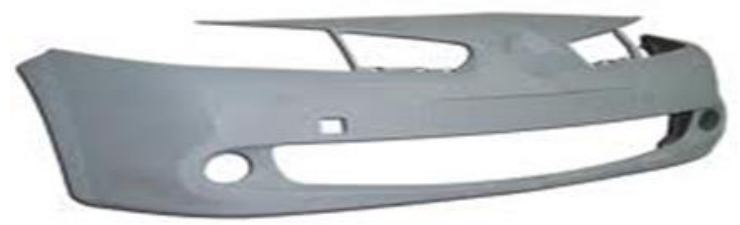

Figura 2. Parachoque

En 1972 el Renault 5 se convirtió en el primer vehículo de gran serie con parachoques de plástico, lo que se generalizó en la década de 1980, siendo más ligeros y seguros. Hoy en día existen diversos materiales para fabricarlos, desde acero o aluminio, hasta caucho y derivados del plástico.

Uno de los logros más significativos en la aplicación de los plásticos, fue la fabricación de depósitos de combustibles con estos materiales, que con procesos de formación mediante insuflado, es posible lograr formas completas que permiten su adaptación a los espacios disponibles en el automóvil y favorece su proceso de diseño.

Desde estos inicios, el uso de los componentes plásticos en automóviles y transporte han experimentado un crecimiento enorme, particularmente durante los últimos 30 años, yendo desde unos pocos kilogramos por coche a casi 105 kilogramos de media por coche construido en el año 2000 .

\section{Panorama actual del uso de los plásticos en la automoción}

En la actualidad, resulta verdaderamente impresionante el trabajo de los fabricantes de automóviles relacionado con el diseño, lo que se evidencia en la gran variedad de modelos que presentan anualmente y que logran satisfacer al usuario más exigente incluso con productos personalizados. Todo esto lleva a la búsqueda y creación de nuevos materiales y técnicas o procesos de fabricación en correspondencia con los criterios actuales de evolución basados en la reducción del peso, el aumento de la resistencia y la seguridad, sin dejar de mencionar los costos de producción.

La ligereza en el peso de los plásticos es un beneficio claro para la industria del automóvil, no sólo porque se consigue reducir el peso total de éstos de modo que se reduce también el consumo de combustible a los límites permitidos por la legislación, sino que también permite realizar sistemas y componentes más sofisticados, tales como sistemas de control de temperatura en el coche y ventilación de este, por no mencionar sistemas adicionales de seguridad como el ya bien conocido airbag.

Mientras la industria automovilística se desarrolla, particularmente, bajo las presiones legislativas que han sido impuestas durante los últimos años, como por ejemplo con relación al consumo de combustible de los autos y el cuidado del medio ambiente, los plásticos están respondiendo de forma satisfactoria en buena medida. Muchísimos costes se están viendo reducidos por la capacidad de los plásticos para ser moldeados en componentes de geometrías complejas, que a menudo reemplazan otras partes construidas con otros materiales, y ofrecen unos ajustes integrales que se añaden a un fácil ensamblaje, lo que ayuda a reducir los costes de la línea de ensamblaje. 
Como dato curioso, cabe decir que se estima que, sin plásticos, los coches de hoy serían entre 200 y $300 \mathrm{~kg}$ más pesados; los resultados del ahorro de gasolina estimado estarían en torno a 0.5 litros a los $100 \mathrm{~km}$, lo que representa unos 750 litros para un coche con una vida de 150 $000 \mathrm{~km}$. Esto conlleva consigo un mayor beneficio si se piensa en la contaminación emitida por cada uno de los coches que se usan.

Teniendo en cuenta los tipos de materiales utilizados en la fabricación de las piezas que componen un vehículo, nos encontraríamos que aproximadamente más de un $70 \%$ del peso del vehículo correspondería a materiales metálicos, y un 14\% serían materiales plásticos. La distribución de estos plásticos según diferentes partes o sistemas en las que se puede dividir un vehículo es aproximadamente la siguiente:

En los acabados interiores se observa el mayor porcentaje de utilización, el plástico es el material mayoritario a la hora de revestir el interior del habitáculo de pasajeros, ejemplos de estas piezas son los revestimientos de puertas, montantes y techo, otras piezas también incluidas dentro del habitáculo son el panel de instrumentos o el acolchado de los asientos. En la fabricación del panel de instrumentos, los plásticos han permitido el diseño de formas complicadas a las que se les concede un papel muy importante a la hora de juzgar la estética del interior del vehículo, y que suponen una línea de marketing para los constructores.

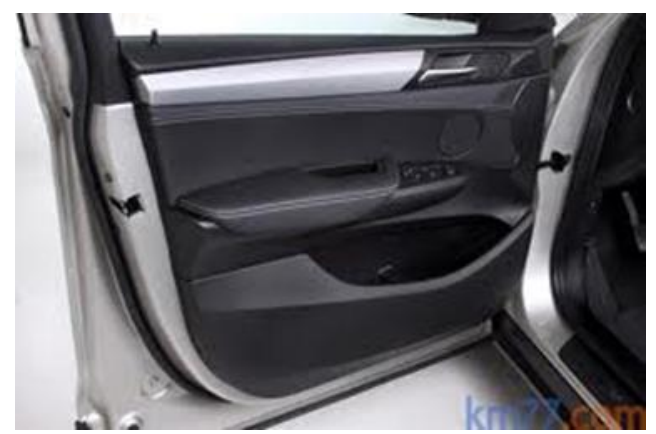

Figura 3.Cobertor interior de puertas

En segundo lugar en cuanto a mayor porcentaje de utilización sería la carrocería, de la cual en la panelería y los acabados externos se podría poner como ejemplos de piezas, a los parachoques, las rejillas, molduras y spoilers, cubiertas de faros, tapacubos y guardabarros, entre otras. La utilización de plástico en la panelería exterior aporta la ventaja frente al acero, de un mejor comportamiento frente a los impactos a baja velocidad y una reducción del peso de las piezas como ya se indicó en otro momento.

Para el caso de los paneles verticales, los plásticos con los que se obtiene un mejor resultado son con los termoplásticos transformados por inyección, con los que se consigue unos buenos acabados superficiales y a un coste razonable. Sin embargo, para los paneles horizontales que necesitan una buena rigidez y un buen comportamiento a temperaturas elevadas, se utilizan los termoestables reforzados con fibra, aunque presentan una mayor fragilidad frente a impactos. En la estructura de la carrocería todavía se necesita una mejora importante en lo referente a los procesos de transformación y unión, así como al coste económico que supone la utilización de materiales adecuados para las piezas estructurales (termoplásticos con fibra transformados por compresión, perfiles de termoestables o termoplásticos extruidos con fibra continua, etc.), la 
utilización de estos materiales todavía es muy cara, en relación con los materiales utilizados hasta ahora, los metales.

\section{Principales plásticos utilizados en el automóvil}

Entre los materiales plásticos más empleados en automoción se encuentran los termoplásticos, los termoestables y los elastómeros (González, 2012).

\section{Termoplásticos}

Están formados por macromoléculas lineales o ramificadas, no entrelazadas. En general son duros, fríos y al calentarlos se reblandecen y fluyen. Sus propiedades mecánicas dependen en gran medida del grado de polimerización y del proceso mecánico de su preparación. Entre los más utilizados tenemos:

\section{ABS (Acrilonitrilo-Butadieno-Estireno):}

Tiene buenas propiedades en cuanto a rigidez, tenacidad, estabilidad dimensional, resistencia a los productos químicos y buena calidad de las superficies. Se emplea en la fabricación de calandras y rejillas, interior del motor, estructuras de salpicaderos, tapacubos, spoilers, cantoneras en estructuras entre otros componentes.

\section{ALPHA (ABS-Policarbonato):}

Propiedades: Presenta buenas propiedades mecánicas y térmicas, es rígido, resistente y con buena estabilidad dimensional.

Usos: spoilers y cantoneras, canalizaciones, rejillas.

\section{PA (Poliamida):}

Propiedades: se fabrica en varias densidades. Es tenaz, resistente al desgaste y a los disolventes usuales.

Usos: rejillas, revestimientos interiores, radiadores, retrovisores.

\section{PC (Policarbonato):}

Propiedades: son materiales rígidos y duros con una excepcional resistencia al impacto. Son dimensionalmente estables, resistentes a la intemperie y al calor.

Usos: parachoques, revestimientos interiores, de posos de rueda.

\section{PE (Polietileno):}

Propiedades: es el polímero de mayor producción. Es resistente a los productos químicos y a las elevadas temperaturas, tiene una gran resistencia a la tracción y al impacto. Es de los mejores eléctricos.

Usos: baterías, paragolpes, revestimientos interiores. 


\section{PP (Polipropileno):}

Propiedades: tiene idénticas aplicaciones que el "PE ad", se comporta mejor que este en altas temperaturas pero peor en las bajas. Es buen aislante y muy resistente a la tracción y a la absorción. Es fácilmente colorearle.

Usos: similares al polietileno. Es el plástico más utilizado en el automóvil.

\section{PP-EPDM (Etileno-propileno-dieno-monómero):}

Propiedades: es elástico y absorbe con facilidad los impactos, es resistente a la temperatura y de buenas propiedades eléctricas. Resiste a los ácidos y disolventes.

Usos: paragolpes, revestimientos interiores y exteriores, spoilers, cantoneras...

\section{PVC (Cloruro de polivinilo):}

Propiedades: resistente a la intemperie y la humedad, pero no a la temperatura, por lo que hay que añadirle diversos estabilizantes. Es dimensionalmente estable, se colorea con facilidad y es resistente a la mayoría de los ácidos. Cuando se descompone, desprende humo tóxico de cloruro de hidrógeno.

Usos: pisos de autocares, cables eléctricos...

\section{XENOY (PC-PBTP):}

Propiedades: aunque de estructura rígida, son elásticos y tienen una gran resistencia al impacto.

Usos: paragolpes, retrovisores, rejillas, revestimientos de pasos de rueda.

\section{Termoestables}

Se denominan así por no sufrir ninguna variación en su estructura al ser calentados; ni se reblandecen ni fluyen al ser sometidos a presión o a calor, siempre que no se llegue a la temperatura de descomposición. Derivados del fenol, su principal propiedad estriba en su resistencia mecánica. Entre los más utilizados tenemos:

\section{GU-P (Resinas de poliéster reforzadas con fibra de vidrio):}

Propiedades: son materiales rígidos, ligeros y de buenas propiedades mecánicas. Usos: portones, capós, isotemos.

\section{GFK (Plásticos reforzados con fibra de vidrio):}

Propiedades: presentan una estructura formada por una resina termo endurecible y fibras de vidrio. Son de una gran fuerza, resistentes a la corrosión y la intemperie y de baja conductibilidad térmica. Las resinas utilizadas pueden ser poliésteres, epoxídicas y fenólicas. 
Debido a que tienen fibras incorporadas, no son soldables pero se pueden reparar. Usos: paragolpes, canalizaciones, salpicaderos.

\section{EP (Epoxi-do) resina epoxi:}

Propiedades: son materiales duros, resistentes a la corrosión y a los agentes químicos, no originan encogimiento.

Usos: se utiliza como adhesivo para los metales y para la mayoría de las resinas sintéticas.

\section{Perspectivas del uso de los plásticos en la automoción}

Es de esperar que el cumplimiento de las regulaciones existentes y sus crecientes niveles de exigencia hará que la industria automotriz continúe realizando esfuerzos por que los plásticos sustituyan partes automotrices en los años futuros. El desarrollo de las tecnologías de los plásticos y el constante mejoramiento de las propiedades de estos, hacen posible un futuro prometedor para estos materiales. La composición química de los plásticos hace que estos sean maleables y de buena manufactura.

Como es de esperar la selección del material dependerá de la función del componente que forme parte del auto, de modo que por un lado habrá componentes que soportarán grandes cargas y otros que cumplirán funciones relativas a la apariencia, garantizar seguridad, cubrir, colocación de instrumentación, organización y confort interior del vehículo, entre otros, de modo que habrá una gama amplia de materiales por elegir. En este sentido existirá la posibilidad de disminuir peso y costos.

Un elemento importante relacionado con la disminución del peso, está en la compactación, condensación y reducción al mínimo indispensable de los órganos mecánicos y otros componentes, incluyendo los fabricados con materiales plásticos. Por otro lado es probable que ambos materiales, metal y plástico deban convivir todavía por largo tiempo siempre y cuando cumplan con la función del componente diseñado.

Se estima que existen actualmente más de 100 tipos de plásticos en partes y componentes de un automóvil y que permiten cumplir con diferentes funciones. Dichas funciones van desde mantener una agradable apariencia, garantizar resistencia mecánica, al impacto, al doblez, a la temperatura y que al mismo tiempo contribuyen a la disminución del vehículo. En el terreno de la ligereza unida a la resistencia, debe continuar desarrollándose la tendencia de combinar metal y plástico para combinar las ventajas de uno y otro material.

Es factible pensar que en un plazo relativamente corto los engranajes en un auto puedan ser en su totalidad fabricados con materiales plásticos, lo que permitirá hacer más productivos y eficientes los procesos de manufactura, así como para la disminución de los costos y aligeramiento del peso del vehículo.

En cuanto a la carrocería, relacionada con los plásticos, hay que tener en cuenta los diversos niveles de exigencia a que son sometidos los elementos horizontales como son el techo, portaequipajes y portezuela posterior, respecto a los verticales tales como el guardabarros y 
frontal. Para cada uno se emplean y deberán emplearse materiales adecuados a las prestaciones requeridas.

Es muy difícil prever el dominio del plástico o del metal por lo que es probable que en cuanto al aumento de la ligereza de un vehículo, ambos materiales deberán contribuir al cumplimiento de este propósito teniendo en cuenta que continuarán desarrollándose las investigaciones para el mejoramiento de las propiedades y prestaciones de ambos tipos de materiales. Por otra parte, la experiencia parece indicar que continuará la supremacía de los materiales plásticos en la construcción de las partes interiores del automóvil, que seguirá estando en la mira como un desarrollo importante para la reducción del peso.

Algunos expertos del sector automotriz son de la opinión de que los automóviles sólo constarán de cuatro a cinco módulos en el futuro. Estos elementos serán muy complejos e que integrarán los componentes más variados en un espacio muy reducido. Sin embargo, la industria del automóvil tiende cada vez más a limitarse en la cantidad de los materiales a utilizar principalmente por razones de costes y medioambientales. Si bien anteriormente se recurría a diversos materiales para un mismo sistema modular, ahora se suele utilizar un solo plástico, como puede ser el polipropileno (PP). En él se ha concentrado la casa Opel. Esto aporta ventajas no sólo en el montaje. También facilita el reciclaje posterior (Tecnología del plástico, 2004).

La industria de plásticos ha demostrado efectivamente que sus termoplásticos están totalmente preparados para ser reciclados por un proceso convencional de fundido, y que sus composites termoestables pueden ser procesados moliéndolos hasta conseguir un polvo que se reutilizará para realizar nuevos compuestos. Para ambos, la industria es rápida desarrollando tecnologías químicas.

No obstante, lo anterior, el mayor desafío para los plásticos en la automoción y transporte será probablemente su reciclabilidad. Aunque la industria de la automoción es posible que tenga el mayor récord de todas las industrias en cuanto se refiere al reciclaje de sus materiales, con una media de alrededor del 75\%, los requerimientos actuales como por ejemplo de la Unión Europea, están establecidos con objetivos mucho más elevados. Lo que si debe estar claro que algo más productivo debe ser hecho con los plásticos que desecharlos en un vertedero (Asociación Española de Industriales de Plástico, 2018).

\section{Conclusiones}

Por todo lo expuesto en al presente artículo se puede afirmar que los plásticos ocupan en la actualidad un lugar preponderante en el sector automotriz, sustituyendo progresivamente y con prestaciones ventajosos a un gran número de materiales empleados en este sector. No cabe duda de que en función de las tendencias actuales en el diseño de automóviles las propiedades de los plásticos deberán seguir satisfaciendo las necesidades de ese importante sector particularmente en lo relativo a la disminución del peso, seguridad y costos.

En la actualidad se estima que la mayoría de los autos comercializables en el mercado internacional poseen un $66 \%$ de sus componentes elaborados con materiales compuestos de matriz polimérica, los cuales son elaborados por los métodos convencionales. Las evidencias demuestran que las ventajas de los plásticos sobre los materiales metálicos, en la rama 
automovilística, les ofertan a estos un mercado próspero y novedoso que augura un y en particular sobre el acero, continuarán influyendo significativamente en el diseño y fabricación de automóviles en el futuro próximo y mediato.

\section{Bibliografía}

(s.f.). Obtenido de http://adriangonzalez-eaf.blogspot.com/2012/04/principales-plasticos-utilizados-enel.html

A. C., E. (2009). Elementos metálicos y sintéticos. Madrid: Paraninfo.

A.G.V.A.G. (2002). Algunas aplicaciones de los plásticos en las industrias del empaque y automotriz.

Asociación Española de Industriales de Plástico. (15 de Noviembre de 2018). Automoción y transporte. Obtenido de https://anaip.es/los-plasticos/aplicaciones/automocion-y-transporte.html

Clavero, D. (07 de 12 de 2012). Diario Motor. Obtenido de https://www.diariomotor.com/tecmovia/2012/12/07/volvo-trucks-ve-posible-la-llegada-de-lostrenes-de-carretera-inteligentes-en-10-anos/

Condezo, C. (15 de Noviembre de 2018). Antecedentes Históricos de los Plásticos. Obtenido de https://www.ebah.com.br/content/ABAAAfUgcAL/antecedentes-historicos-los-plasticos

Ezio, M. (1993). La materia de la invención. Barcelona: Grupo Editorial Ceac.

González, A. (25 de Mayo de 2012). Elementos amovibles y fijos. Obtenido de http://adriangonzalezeaf.blogspot.com/2012/04/principales-plasticos-utilizados-en-el.html

INEN. (2002). Gestión Ambiental, Aire, Vehículos Automotores. Quito: INEN.

Martínez, E., \& Díaz de Mera Morales, Y. (2014). Contaminación atmosférica. Universidad de Castilla-La Mancha, 13. Obtenido de Contaminación atmosférica.

Muñoz. (1998). Tendencias actuales del plástico en la manufactura. Madei: pertr.

Nitro Química. (15 de noviembre de 2018). Nitro celulosa Manual Técnico de Aplicación. Obtenido de http://www.salazarsa.com/Nitrocelulosa-manual_tecnico_de_aplicacion-parte1.pdf

Schmid, S. (2008). Manufactura, ingeniería y tecnología. México: Prentice Hall.

Tecnalia. (03 de 02 de 2012). Tecnalia. Obtenido de https://www.tecnalia.com/es/industriatransporte/noticias/proyecto-sartre-conduccion-autonoma.htm

Tecnología del plástico. (Noviembre de 2004). El nuevo papel de los plásticos en la industria automotriz. Obtenido de http://www.plastico.com/temas/El-nuevo-papel-de-los-plasticos-en-la-industriaautomotriz+3034780

Tecnum. (2002). Ciencias de la tierra, Sustancias que contaminan la atmósfera. Gipuzkoa: Tecnun. 